\title{
COMMUTATIVE RINGS WITH COMPARABLE REGULAR ELEMENTS
}

\author{
PAOLO ZANARDO \\ (Received 21 June 1993; revised 24 January 1994) \\ Communicated by P. Schultz
}

\begin{abstract}
Let $\mathscr{R} \mathscr{C}$ be the class of commutative rings $R$ with comparable regular elements, that is, given two non zero-divisors in $R$, one divides the other. Applying the notion of $V$-valuation due to Harrison and Vitulli, we define the class $V$-val of $V$-valuated rings, which is contained in $\mathscr{R} \mathscr{C}$ and contains the class of Manis valuation rings. We prove that these inclusions of classes are both proper. We investigate Prüfer rings inside $\mathscr{R} \mathscr{C}$, showing that there exist Prüfer rings which lie in $\mathscr{R} \mathscr{C}$ but not in $V$-val; we prove that a ring $R$ is a Prüfer valuation ring if and only if it is Prüfer and $V$-valuated, if and only if its lattice of regular ideals is a chain. Finally, we introduce and investigate the ideal $I_{\infty}$ of a ring $R \in \mathscr{R} \mathscr{C}$, which corresponds to the counterimage of $\infty$, whenever $R$ is $V$-valuated.
\end{abstract}

1991 Mathematics subject classification (Amer. Math. Soc.): 13A18, 13 F30.

\section{Introduction}

The classical notion of a valuation of a field, given by Krull in his fundamental paper [9], was extended by Manis to commutative rings [10]. More recently, Harrison and Vitulli introduced in [4] the notion of $V$-valuation of a commutative ring, which includes the preceding ones; in their papers $[4,5,6]$, they were mainly interested in investigating rings (possibly integral domains) through the various $V$-valuations defined on them.

Adopting a different point of view, the present paper is devoted to investigate classes $\mathscr{A}$ of commutative rings which satisfy properties that force an $R \in \mathscr{A}$ to be a valuation domain, whenever $R$ is an integral domain. The largest possible of these classes, which we denote by $\mathscr{R} \mathscr{C}$, is constituted by commutative rings $R$ with comparable regular elements, which means that, for any given $a, b \in R$ regular, that

Lavoro eseguito con il contributo del MURST.

(C) 1996 Australian Mathematical Society 0263-6115/95 \$A2.00+0.00 
is non zero-divisors, we have that one of them is a multiple of the other.

We call a ring $R V$-valuated if there is a $V$-valuation $v$ defined on the total ring of fractions $T(R)$ of $R$, such that $R=\{x \in T(R): v(x) \geq 0\}$; of course, this definition agrees with the classical one of valuation domain, and also with that of Manis valuation ring (see [7]). For a ring $R$ to be $V$-valuated is equivalent to a very simple property: namely $(T(R) \backslash R)(T(R) \backslash R) \subseteq T(R) \backslash R$; the idea of investigating a subring whose complement is multiplicatively closed, as an extension of the notion of valuation domain, goes back to Samuel [14].

The class $\mathscr{R} \mathscr{C}$ contains the class $V$-val of $V$-valuated rings, and $V$-val contains the class $\mathscr{M} \mathscr{V} \mathscr{R}$ of Manis valuation rings.

In the first section we give some easy properties of $V$-valuated rings, and show in Theorems 1.7 and 1.8 that the inclusions $\mathscr{R} \mathscr{C} \supset V$-val $\supset \mathscr{M} \mathscr{V} \mathscr{R}$ are all proper (we note that our Theorem 1.8 follows also from the results by Gräter [3] or Lucas [8]).

In the second section we examine the rings of $\mathscr{R} \mathscr{C}$ through properties of their lattice of ideals. We fix our attention in particular on Prüfer rings; these rings, which extend the classical notion of Prüfer domains, have raised considerable interest in recent years (for a large list of references see the book by Huckaba [7]). We prove that there exist Pruifer rings in $\mathscr{R} \mathscr{C}$ which are not $V$-valuated (Theorem 2.1); on the other hand, if a Prüfer ring is $V$-valuated, then it is automatically a Manis valuation ring. This result is acheived, in Theorem 2.9, through a characterization of Prüfer $V$-valuated rings in terms of the lattice of their regular ideals (that is ideals which contain regular elements): a ring $R$ is a Prüfer $V$-valuated ring if and only if it is a Prüfer valuation ring if and only if the lattice of its regular ideals is a chain (this condition implies that $R \in \mathscr{R} \mathscr{C}$ ). Let us recall that the second equivalence of the preceding result had been already proved by Anderson and Pascual in [2].

In the third section, for a given $R \in \mathscr{R} \mathscr{C}$, with $R \neq T(R)$, we define and investigate the ideal $I_{\infty}$, which, when $R$ is $V$-valuated by the $V$-valuation $v$, corresponds to the prime ideal $v^{-1}(\infty)$, the counterimage of $\infty$. In particular, we show in Example 3.5 that $I_{\infty}$ is not prime, in general, if $R \in \mathscr{R} \mathscr{C} \backslash V$-val.

We finally remark that all our constructions of rings in $\mathscr{R} \mathscr{C}$ are based on the method of idealization, originally due to Nagata [13].

The author is grateful to the referee for a number of suggestions, improvements and corrections to the original manuscript.

In the sequel, every ring considered will be commutative with identity. Let $R$ be a ring; an element $r$ in $R$ is said to be regular if it is not a zero-divisor; an ideal $I$ of $R$ is said to be regular if $I$ contains regular elements. We denote by $Z(R), \operatorname{Reg} R$ and 
$U(R)$ the sets of zero-divisors, of regular elements and of units of $R$, respectively; the total ring of fractions of $R$ is denoted by $T(R)$. If an ideal $I$ of $R$ is generated by the subset $X \subseteq R$, we shall write, as usual, $I=(X)$.

Two regular elements $a, b \in R$ are said to be comparable if either $a$ divides $b$ in $R$, or viceversa. We say that a ring $R$ has comparable regular elements if, for any assigned $a, b \in \operatorname{Reg} R, a$ and $b$ are comparable; we denote by $\mathscr{R} \mathscr{C}$ the class of rings which satisfy this property (the class $\mathscr{R} \mathscr{C}$ was already considered in [16]).

LEMMA 1.1. If $R \in \mathscr{R} \mathscr{C}$, then the ideal of $R$ generated by $\operatorname{Reg} R \backslash U(R)$ is proper.

PROOF. It is enough to prove that no $x$ in the ideal $(\operatorname{Reg} R \backslash U(R))$ is a unit. In fact, we have $x=\sum \lambda_{i} a_{i}$, for suitable $a_{i} \in \operatorname{Reg} R \backslash U(R)$ and $\lambda_{i} \in R$; since $R \in \mathscr{R} \mathscr{C}$, one of the $a_{i}$, say $a_{1}$, divides all the others in $R$; therefore $x=a_{1}\left(\sum \lambda_{i} a_{i} / a_{1}\right)$, and from $a_{1} \notin U(R)$ it follows $x \notin U(R)$, as desired.

Of course, the statement of Lemma 1.1 is not invertible, as we can see by easy examples.

In the paper [16] it is shown that the class $\mathscr{R} \mathscr{C}$ properly contains the class $\mathscr{M} \mathscr{V} \mathscr{R}$ of Manis valuation rings; the book by Huckaba [7] is our main reference for definitions and results about valuations on commutative rings.

Harrison and Vitulli introduced in [4] the notion of $V$-valuation of a commutative ring $S$. It is convenient to recall their main definitions (see Definitions 2.1 and 2.10 of [4]).

DEFINITION H-V 1. A $V$-monoid is a triple $(\Gamma,+, \leq)$ such that:

(i) $(\Gamma,+)$ is a commutative monoid.

(ii) $(\Gamma, \leq)$ is a totally ordered set with a maximum denoted by $\infty$.

(iii) For all $\alpha, \beta, \gamma$ in $\Gamma$,

$$
\alpha \leq \beta \Rightarrow \alpha+\gamma \leq \beta+\gamma
$$

(iv) For all $\gamma \in \Gamma$,

$$
\gamma+\infty=\infty
$$

(v) For all $\alpha, \beta \in \Gamma$,

$$
\alpha<\beta \Rightarrow \exists \gamma \in \Gamma \text { such that } \alpha+\gamma<0 \leq \beta+\gamma .
$$

From the above definition of $V$-monoid it follows also that, for $\alpha, \beta, \gamma, \delta \in \Gamma$, $\alpha<\beta$ and $\gamma<\delta$ implies $\alpha+\gamma<\beta+\delta$, whence, in particular, $\alpha, \beta<\infty$ implies $\alpha+\beta<\infty$ (see [4, Lemma 2.5]). On the other hand $\alpha<\beta$ and $\gamma<\infty$ does not guarantee $\alpha+\gamma<\beta+\gamma$, in general. 
DEFINITION H-V 2 A $V$-valuation of a commutative ring $R$ is a pair $(v, \Gamma)$, where $\Gamma$ is a $V$-monoid and $v: R \rightarrow \Gamma$ is a surjective map, such that:

(a) $v(r s)=v(r)+v(s)$ for all $r, s \in R$;

(b) there exists a unit $t \in R$ such that:

(i) $\min \{v(r), v(s)\} \leq v(r+s)+v(t)$ for all $r, s \in R$;

(ii) $v(s)<0 \Rightarrow \exists n \in \mathbb{N}$ such that $n v(s)+v(t)<0$.

We note at once that the definition of $V$-valuation become simplified in the case in which we are interested.

We must, however, slightly enlarge the notion of $V$-monoid given in Def. H-V 1; namely, we require that $\Gamma=\{0, \infty\}$, with the obvious structure, be a $V$-monoid (note that $\{0, \infty\}$ does not satisfy condition (v) in Def. H-V 1$)$. This assumption is needed to get rid of the apparently trivial case when a ring $R$ coincides with its total ring of fractions $T(R)$.

DEFINITION. A ring $R$ is $V$-valuated if there exists a $V$-valuation $v$, defined on the total ring of fractions $T(R)$ of $R$, such that $R=R_{v}=\{x \in T(R): v(x) \geq 0\}$.

The above definition is the most natural, because if a $V$-valuated ring is a domain, then $R$ is a valuation domain, in the usual sense; also, a $V$-valuated ring $R$ is a Manis valuation ring if and only if the $V$-monoid $\Gamma$ is of the form $\Gamma=G \cup\{\infty\}$, where $G$ is a totally ordered abelian group.

The following facts about $V$-valuated rings are straightforward consequences of Definition H-V 2, Definition 2.13, and Theorem 2.15 of [4].

PROPOSITION 1.2. Let $R$ be a ring with total ring of fractions $T=T(R)$. Then $R$ is $V$-valuated if and only if

$$
(T \backslash R)(T \backslash R) \subseteq T \backslash R
$$

We note that Samuel [12] already observed that a domain satisfying $(T \backslash R)(T \backslash R) \subseteq$ $T \backslash R$ is a valuation domain.

Let $R$ be $V$-valuated and suppose that $R \neq T(R)$. Then the $V$-valuation defined on $T=T(R)$ such that $R=R_{v}$ is unique up to isomorphism ([4], Theorem 2.15); it is given by

$$
x \mapsto v(x)=(R: x)=\{t \in T: t x \in R\} .
$$

The sum on the $V$-monoid $\Gamma=\{v(x)=(R: x): x \in T\}$ is defined by $v(x)+v(y)=$ $v(x y)$, and the total order on $\Gamma$ by

$$
v(x) \leq v(y) \Longleftrightarrow(R: x) \subseteq(R: y) .
$$


We have $v(1)=0$ (neutral element of $\Gamma$ ) and $v(0)=\infty$. In our situation $t=1$ satisfies (b) of Def. H-V 2 (see [4, Remark 2.14]); therefore the $V$-valuation $v$ satisfies the two usual properties enjoyed by valuations:

$$
\begin{aligned}
& v(x y)=v(x)+v(y) \\
& v(x+y) \geq \min \{v(x), v(y)\} \quad \text { for all } x, y \in T .
\end{aligned}
$$

Let us also observe that, if $r \in \operatorname{Reg} R$, then $(R: r)=1 / r R$; hence, if $R$ is $V$-valuated and $x \in T(R)$, then $v(x)<v(r)$ if and only if $x / r \notin R$; in particular, $v(r)<v(r s)$, for all $r \in \operatorname{Reg} R$ and $s \in \operatorname{Reg} R \backslash U(R)$.

Let us now look at the case when $R$ coincides with $T(R)$.

Proposition 1.3. Let $R$ be a ring such that $R=T(R)$. Then $R$ is a Manis valuation ring, and there is a one-to-one correspondence between the prime ideals $\mathscr{P}$ of $R$ and the valuations of $R$; in any case the image of a valuation of $R$ is the $V$-monoid $\Gamma=\{0, \infty\}$.

PROOF. For a given prime ideal $\mathscr{P}$ of $R$ we define a map $v_{\mathscr{P}}: R \rightarrow\{0, \infty\}$ by setting, for $x \in R, v_{\mathscr{P}}(x)=0$ if $x \notin \mathscr{P}, v_{\mathscr{P}}(x)=\infty$ if $x \in \mathscr{P}$; since $\mathscr{P}$ is prime, we readily see that $v_{\mathscr{P}}$ satisfies properties (i) and (ii) above, hence it is a valuation. Vice-versa, for a given valuation $v$ of $R$, the prime ideal $\mathscr{P}=v^{-1}(\infty)$ is such that $v=v_{\mathscr{P}}$. Finally, note that $T(R)=R=\{x \in T(R): v(x) \geq 0\}$, implies that $v(R)=\{0, \infty\}$, in any case.

We have thus seen that $R$ does not determine its $V$-valuation, if $R=T(R)$.

In the third section we shall need the following property of a $V$-valuated ring (cf. [1, Lemma 1.1]).

Proposition 1.4. Let $R \neq T(R)$ be $V$-valuated and let $v$ be the $V$-valuation defined on $T(R)$. For all $x \in R$ such that $v(x)<\infty$ there exists $r \in \operatorname{Reg} R$ such that $v(x)<v(r)$.

PROOF. We use property (v) of Def. H-V 1: given $x \in R$ with $0 \leq v(x)<\infty$, there exists $\xi \in T(R)$ such that

$$
v(x)+v(\xi)<0<\infty .
$$

We have $\xi=z / r$ with $z \in R, r \in \operatorname{Reg} R$; hence $v(x)+v(z)+v(1 / r)<0$. From $v(z) \geq 0$ and (iii) of Def. H-V 1, we get $v(x / r)=v(x)+v(1 / r)<0$; as observed above, $v(x / r)<0$ implies $v(x)<v(r)$, as desired.

We shall denote by $V$-val the class of $V$-valuated rings. 
Propostrion 1.5. If $R$ is $V$-valuated, then $R \in \mathscr{R} \mathscr{C}$.

Proof. By contradiction, let us suppose that $a, b \in \operatorname{Reg} R$ are not comparable; hence $a / b$ and $b / a$ are both in $T=T(R)$ but not in $R$. Then $(a / b)(b / a)=1 \in R$ implies that $(T \backslash R)(T \backslash R) \nsubseteq T \backslash R$, whence $R$ is not $V$-valuated which is impossible.

Thus we have the following inclusions of classes:

$$
\mathscr{R} \mathscr{C} \supset V \text {-val } \supset \mathscr{M} \mathscr{V} \mathscr{R}
$$

moreover $\mathscr{R} \mathscr{C} \supsetneqq \mathscr{M} \mathscr{V} \mathscr{R}$, in view of [16, Theorem 6].

We note that the proper inclusion $V$-val $\supsetneqq \mathscr{M} \mathscr{V} \mathscr{R}$ is not an obvious consequence of our definitions. In [4, Theorem 3.14] $V$-valuations are constructed with values into $V$-monoids $\Gamma$ which are not of the form $G \cup\{\infty\}$, with $G$ a totally ordered abelian group; however, these $V$-valuations are defined on rings which are integral domains and so they do not fall in the case of $V$-valuated rings. Our next purpose is to show that the inclusions in $\left({ }^{*}\right)$ are both proper.

All our constructions of rings will be based on the method of idealization, which was already extensively used in [16]. We refer to Chapter VI of [7] for the results on idealizations which we shall invoke henceforth. We recall the definition, given by Nagata in [13]. Let $R$ be a ring, $B$ an $R$-module; the idealization of $B$ in $R$ is the ring, denoted by $R(+) B$, whose elements are the couples $(r, b), r \in R, b \in B$, endowed with componentwise sum, and product defined by

$$
\left(r, b_{1}\right)\left(s, b_{2}\right)=\left(r s, r b_{2}+s b_{1}\right) .
$$

We shall consider $R$ a subring of $R(+) B$, via the embedding $r \mapsto(r, 0)$.

The following lemma relates idealizations and rings in $\mathscr{R} \mathscr{C}$.

LEMMA 1.6. Let $R$ be a ring, $B$ an $R$-module, and let

$$
\mathscr{X}_{R}=\{r \in R: r \in \operatorname{Reg}(R(+) B)\} \subseteq \operatorname{Reg} R .
$$

Then $R(+) B \in \mathscr{R} \mathscr{C}$ if and only if the elements of $\mathscr{X}_{R}$ are comparable in $R$ and $r B=B$ for all $r \in \mathscr{X}_{R}$.

PROOF. $(\Longleftarrow)$ It is routine to prove that

$$
\operatorname{Reg}(R(+) B)=\left\{(r, b): r \in \mathscr{X}_{R}, b \in B\right\}
$$

(see [7, Theorem 25.3]). Let $(r, b),\left(s, b^{\prime}\right) \in \operatorname{Reg}(R(+) B)$ with $r, s \in \mathscr{X}_{R}$, where we assume, without loss, that $s=r t$ with $t \in R$; then we have

$$
(r, b)(t, x)=\left(s, b^{\prime}\right)
$$


where $x \in B$ is such that $r x=b^{\prime}-b t$, possible, since $r B=B$. Therefore $(r, b)$ and $\left(s, b^{\prime}\right)$ are comparable; from the arbitrariness of their choice we get that $R(+) B \in \mathscr{R} \mathscr{C}$.

$(\Longrightarrow)$ From $R(+) B \in \mathscr{R} \mathscr{C}$ it follows that any two elements $r, s$ of $\mathscr{X}_{R}$ are comparable in $R$, since $(r, 0)$ and $(s, 0)$ are comparable in $R(+) B$. Fix now $r \in \mathscr{X}_{R}$ and let us prove that $B=r B$. In fact, for all $b \in B$, the elements $(r, b)$ and $(r, 0)$ are in $\operatorname{Reg}(R(+) B)$ and so they are comparable; assume first that $(r, b)$ divides $(r, 0)$. Then $(r, 0)=(r, b)(u, x)$, for suitable $u \in R, x \in B$, that is $r=r u, 0=r x+u b$, from which we get $u=1$ (since $r \in \operatorname{Reg} R$ ) and therefore $b=-r x$ is divisible by $r$ in $B$. Analogously, if $(r, 0)$ divides $(r, b)$, we get that $b$ is again divisible by $r$ in $B$. The desired conclusion follows.

THEOREM 1.7. There exist rings $R$ in $\mathscr{R} \mathscr{C}$ which are not $V$-valuated.

ProOf. Let $A$ be a valuation domain with maximal ideal $P$ and field of quotients $Q, x$ an indeterminate, and let us consider the following subring of $A[x]$ :

$$
D=A+x P+x^{2} A[x]
$$

consisting of those polynomials such that the coefficient of $x$ lies in $P$. Set

$$
\mathscr{U}=\{f \in Q[x]: f \text { irreducible }\} ; \quad B=\bigoplus_{f \in \mathscr{U}} Q[x] /(f)
$$

and consider the idealization $R=D(+) B$. We shall prove that $R \in \mathscr{R} \mathscr{C} \backslash V$-val. If $g(x) \in D$ is a non constant polynomial then $g(x) \in(f)$ for some irreducible polynomial $f \in Q[x]$, whence

$$
(g(x), 0)(0,1+(f))=(0,0)
$$

so that $g(x) \in Z(D(+) B)$. Thus we easily conclude that $\mathscr{X}_{D}=A \backslash\{0\}$. Since $A$ is a valuation domain, the elements of $\mathscr{X}_{D}$ are comparable in $D \supset A$; it is also obvious that $a B=B$ for all $a \in A$. Therefore we can apply Lemma 1.6 to get $R \in \mathscr{R} \mathscr{C}$. Moreover $R \notin V$-val; in fact, for any $0 \neq p \in P$ we have $p x / p=x \in T(R) \backslash R$, while $x^{2} \in D \subset R$ so that $(T(R) \backslash R)(T(R) \backslash R) \nsubseteq T(R) \backslash R$. The desired conclusion follows.

As already observed in the introduction, our next theorem is a consequence of the results by Gräter [3] or Lucas [8], which both constructed paravaluation rings (see [7]) of their total ring of fractions which are not valuation rings; such paravaluation rings are automatically $V$-valuated. To prove Theorem 1.8 we make use of a ring in $\mathscr{R} \mathscr{C}$ which already appeared in [16, Theorem 6]. 
THEOREM 1.8. There exist $V$-valuated rings which are not Manis valuation rings.

PROOF. Part of the proof is contained in that of [16, Theorem 6]; in fact we consider the same ring examined there (with a change of notation); let us now recall its definition. Let $A$ be a valuation domain with Krull dimension $>1$, so that $A$ contains a nonzero prime ideal $P_{1}$ different from the maximal $P$. Set

$$
D=A+x P_{1}[x] ; \quad B=\bigoplus_{f \in \mathscr{U}} Q[x] /(f) ; \quad R=D(+) B
$$

where $P_{1}[x]$ are the polynomials with coefficients in $P_{1}$ and $Q, \mathscr{U}$ are as in the proof of Theorem 1.7. It is proved in Theorem 6 of [16] that $R \in \mathscr{R} \mathscr{C} \backslash \mathscr{M} \mathscr{V} \mathscr{R}$. To conclude, it is enough to show that $(T \backslash R)(T \backslash R) \subseteq T \backslash R$, where $T=T(R)$. As in the proof of Theorem 1.7, we see that $\mathscr{X}_{D}=A$; using the fact that $(1, b)$ is a unit of $R$ for all $b \in B$ and $a B=B$ for all $0 \neq a \in A$, one readily checks that

$$
T=\{(f(x) / a, b): 0 \neq a \in A, f(x) \in D, b \in B\}
$$

(cf. Corollary 25.5 of [7]). To verify that $(T \backslash R)(T \backslash R) \subseteq T \backslash R$ it is enough to prove that if $f(x) / a, g(x) / a^{\prime} \in Q[x] \backslash D$, with $f(x), g(x) \in D, a, a^{\prime} \in A$, then $f(x) g(x) / a a^{\prime} \notin D$. It is convenient to distinguish two different cases:

(I) let $f(x) / a=1 / p+x \phi(x), g(x) / a^{\prime}=1 / q+x \psi(x)$, with $1 / p, 1 / q \in Q \backslash A$, $\phi(x), \psi(x) \in Q[x]$; it is then clear that $f(x) g(x) / a a^{\prime} \notin A[x] \supset D$.

(II) If we are not in case (I), we can assume without loss that $f(x) / a=\alpha+x \phi(x)$, with $\alpha \in A$ and $\phi(x) \in Q[x] \backslash P_{1}[x]$. If $\lambda \in A$ is the least common multiple of the denominators of the coefficients of $\phi(x)$ (recall that $A$ is a valuation domain), it is easy to see that $\lambda f(x) / a \in A[x] \backslash D$ so that $\lambda f(x) / a=\sum f_{i} x^{i}$, where $f_{i} \in A$ and there exists a maximal $h \geq 1$ such that $f_{h} \notin P_{1}$. In a similar way, we can multiply $g(x) / a^{\prime}$ by a suitable $\mu \in A$ in such a way that $\mu g(x) / a^{\prime}=\sum g_{j} x^{j} \in A[x]$ and there exists a maximal $k$ such that $g_{k} \notin P_{1}$ (here we cannot ask that $k \geq 1$ ). Let us show that $\lambda f(x) \mu g(x) / a a^{\prime} \notin D$, so that a fortiori $f(x) g(x) / a a^{\prime} \notin D$. Since $h+k \geq 1$, it suffices to show that the coefficient of $x^{h+k}$ in $\lambda f(x) \mu g(x) / a a^{\prime}$ is not an element of $P_{1}$; actually this coefficient is

$$
\sum_{i+j=h+k} f_{i} g_{j}=f_{h} g_{k}+\rho
$$

where $f_{h} g_{k} \notin P_{1}$, while $\rho \in P_{1}$ in view of the maximality of $h, k$.

We have thus proved our assertion.

It could be interesting to examine the $V$-monoid $\Gamma$ associated to the $V$-valuated ring $R$ in the proof above. One sees, for instance, that the element $v(x) \in \Gamma$ has no opposite element in $\Gamma$, and that $v(1 / q)<v(x)<v(1 / p)<0$ for all nonzero $p \in P \backslash P_{1}$ and $q \in P_{1}$. 
The main purpose of this section is to investigate Prüfer rings inside the class $\mathscr{R} \mathscr{C}$.

Recall that a ring $R$ is said to be a Prüfer ring if every regular finitely generated ideal $J$ of $R$ is invertible, that is $J J^{-1}=R$, where $J^{-1}=(R: J)=\{\xi \in T(R): \xi J \subseteq R\}$ (see [7], in particular Theorem 6.2 for equivalent definitions of Prüfer rings).

A first natural question is if there are Prüfer rings in $\mathscr{R} \mathscr{C}$ but not in $V$-val; this question is also motivated by [7, Theorem 7.7] in the sense we now explain.

Recall that a ring $R$ is called a Marot ring if every regular ideal of $R$ is generated by regular elements $[12,7]$. When dealing with valuations the class of Marot rings is a natural subclass of the one of Prüfer rings.

If $R$ is a Marot ring, then $\mathrm{R}$ is in $\mathscr{R} \mathscr{C}$ if and only if $R$ is a Manis valuation ring (and in this case it is also a Prüfer valuation ring) [7, Theorem 7.7]. Thus a Prüfer ring $R \in \mathscr{R} \mathscr{C} \backslash V$-val shows that the above result cannot be extended from Marot to Prüfer rings.

In the next results we shall make free use of some notions about Dedekind domains, for which we refer to the book by Marcus [11]. If $D_{0} \subset D$ are Dedekind domains, we shall always assume that the field of quotients of $D$ is an algebraic extension of the one of $D_{0}$; if $\alpha \in D$, we denote by $N_{D_{0}}^{D}(\alpha)$ the relative norm of $\alpha$. If $\mathscr{M}$ is a prime ideal of $D$, we denote by $D_{\mathscr{M}}$ the localization of $D$ at $\mathscr{M}$.

THEOREM 2.1. Let $D_{0} \subset D$ be Dedekind domains, where $D_{0}$ is a unique factorization domain; let $p$ be a prime element of $D_{0}$ satisfying the following properties:

(a) there exist $\alpha_{1}, \alpha_{2} \in D$ such that $\alpha_{1}, \alpha_{2} \notin p D$ and $\alpha_{1} \alpha_{2} \in p^{2} D$;

(b) if $p^{k}=N_{D_{0}}^{D}(\beta)$, for suitable $k \in \mathbb{N}$ and $\beta \in D$, then necessarily $\beta$ is of the form $\beta=p^{h} u$ with $h \in \mathbb{N}$ and $u$ a unit of $D$.

Set

$$
\mathscr{U}=\{\mathscr{M}: \mathscr{M} \text { prime ideal and } p \notin \mathscr{M}\} ; \quad B=\bigoplus_{\mathscr{M} \in \mathscr{U}} D_{\mathscr{M}} / \mathscr{M}
$$

Then the idealization $R=D(+) B$ is a Prüfer ring which lies in $\mathscr{R} \mathscr{C}$ and is not $V$-valuated.

PROOF. In order to verify that $R \in \mathscr{R} \mathscr{C}$, let us look for $\mathscr{X}_{D}$ (notation of Lemma 1.6). First of all, $p$ is a unit in any $D_{\mathscr{M}}$, since $p \notin \mathscr{M}$ for all $\mathscr{M} \in \mathscr{U}$ and this yields $p \in \mathscr{X}_{D}$ and $B=p B$. If $\alpha \in D$ lies in some prime ideal $\mathscr{M} \in \mathscr{U}$, then $\alpha \in Z(R)$, since $(\alpha, 0)(0,1+\mathscr{M})=(0,0)$; otherwise $\alpha D=\mathscr{P}_{1} \ldots \mathscr{P}_{t}$, where $p \in \mathscr{P}_{i}$ for $1 \leq i \leq t$. This yields $\alpha D \gamma D=p^{k} D$, for suitable $\gamma \in D, k \in \mathbb{N}$, so that $N_{D_{0}}^{D}(\alpha)$ is a power of $p$ times a unit of $D$, whence, in view of (b), $\alpha=p^{h} u$ for suitable $h \in \mathbb{N}$ and $u \in U(D)$. Thus we deduce that $\mathscr{X}_{D}=\left\{p^{k} u: k \in \mathbb{N}, u \in U(D)\right\}$; it is then obvious 
that the elements of $\mathscr{X}_{D}$ are comparable in $D$. Moreover, from $B=p B$ it follows that $r B=B$ for all $r \in \mathscr{X}_{D}$; therefore $R \in \mathscr{R} \mathscr{C}$ by Lemma 1.6.

The fact that $R \notin V$-val is an immediate consequence of (a); in fact, $\alpha_{1} / p, \alpha_{2} / p \in$ $T(R) \backslash R$ and $\alpha_{1} \alpha_{2} / p^{2} \in D \subset R$, so that $(T(R) \backslash R)(T(R) \backslash R) \nsubseteq T(R) \backslash R$.

It remains to check that $R$ is a Prüfer ring. Now $D$ is a Prüfer domain, being a Dedekind domain; this suffices to ensure that the idealization $R=D(+) B$ is Prufer, too [7, Corollary 25.12].

We have to show the existence of $D_{0}, D, p$ satisfying the hypotheses of Theorem 2.1. We remark that $D_{0}, D$ cannot be ring of integers in suitable number fields, due to the fact that the class group of these kind of Dedekind domains is finite. The author is indebted to $U$. Zannier for having suggested to him the two next results.

LEMMA 2.2. In the notation of Theorem 2.1, assume that $p D=\mathscr{P}_{1} \mathscr{P}_{2}$, where the $\mathscr{P}_{i}$ are prime ideals of $D$ whose isomorphism classes have infinite orders in the class group $\mathscr{C}(D)$ of $D$; then p satisfies condition (b).

PROOF. Let us suppose that $k \in \mathbb{N}$ and $\beta \in D$ are such that $N_{D_{0}}^{D}(\beta)=p^{k}$. Then $p^{k}=\beta \bar{\beta}$, where $\bar{\beta} \in D_{0}[\beta] \subset D$; therefore we have the following equality of ideals of $D$

$$
p^{k} D=\beta D \bar{\beta} D .
$$

The uniqueness of factorization implies that $\beta D=\mathscr{P}_{1}^{m} \mathscr{P}_{2}^{n}$, for suitable $m, n \in \mathbb{N}$, where we assume that $m-n=h \geq 0$, so that $\beta D=p^{n} \mathscr{P}_{1}^{h}$. Consequently $\mathscr{P}_{1}^{h}$ is a principal ideal, whence, necessarily, $h=0$ since the isomorphism class of $\mathscr{P}_{1}$ has infinite order in $\mathscr{C}(D)$. Thus $\beta D=p^{n} D$, whence $\beta=p^{n} u$ for a suitable $u \in U(D)$. We conclude that condition (b) is satisfied.

In the next proposition we take for granted some known results on the nonsingular elliptic curve of equation $y^{2}=f(x)$, where $f(x)$ is a polynomial of degree 3 in $\mathbb{C}[x]$ with no multiple roots. A description of the results we invoke may be found in the book by Silverman [15]; we just recall that the group $P i c_{0}$ defined on the elliptic curve is full of non-torsion elements, since it is isomorphic to $\mathbb{C} / \Lambda, \Lambda$ a lattice.

PROPOSITION 2.3. Let $x$ be an indeterminate over the complex field $\mathbb{C}$. Let $D_{0}=$ $\mathbb{C}[x], D=D_{0}[y]$ where $y^{2}=f(x)$ is the equation of a nonsingular elliptic curve. Let us choose $x_{0} \in \mathbb{C}$ in such a way that the point $A=\left(x_{0}, y_{0}\right)$, where $y_{0}^{2}=f\left(x_{0}\right)$, is not torsion in the group Pic $c_{0}$ on the elliptic curve and let $p=x-x_{0} \in D_{0}$. Then $D_{0}$, $D$ and $p$ satisfy the hypotheses of Theorem 2.1. 
PROOF. Let us note $y_{0} \neq 0$, since $A$ is not torsion. In the Dedekind domain $D$ the ideal $p D$ factorizes as follows:

$$
p D=\mathscr{P}_{1} \mathscr{P}_{2} ; \quad \mathscr{P}_{i}=\left(x-x_{0}\right) D+\left(y \pm y_{0}\right) D .
$$

The points $A=\left(x_{0}, y_{0}\right)$ and $A^{\prime}=\left(x_{0},-y_{0}\right)$ are both non-torsion in the group $P i c_{0}$, which is isomorphic to the class group $\mathscr{C}(D)$ of $D$; then the isomorphism classes of $\mathscr{P}_{1}, \mathscr{P}_{2}$, corresponding to $A, A^{\prime}$ respectively, have infinite order in $\mathscr{C}(D)$. Therefore $p$ satisfies (b), in view of Lemma 2.2. Let us now check that $p$ satisfies (a) too. We set $\alpha_{1}=a(x)+y, \alpha_{2}=a(x)-y \in D$, where $a(x) \in \mathbb{C}[x]$. To have $\alpha_{1}, \alpha_{2} \notin p D$ and $\alpha_{1} \alpha_{2} \in p^{2} D$ it is enough that

$$
a\left(x_{0}\right) \neq 0 ; \quad\left(x-x_{0}\right)^{2} \text { divides } a^{2}(x)-f(x) .
$$

Using the fact that $f\left(x_{0}\right) \neq 0$ (and $\mathbb{C}$ is algebraically closed), we can see, by an easy exercise, that (2) is verified with $a(x)$ a suitable polynomial of degree one.

We now look for informations on rings in $\mathscr{R} \mathscr{C}$ by examining their lattice of ideals. For a given commutative ring $A$, we denote by $L(A)$ the lattice of the ideals of $A$. Let $\mathscr{L}$ be the class of lattices $L$ such that $L \cong L(A)$, for some ring $A$.

PROPOSITION 2.4. For every $L \in \mathscr{L}$ there exists $R \in \mathscr{R} \mathscr{C}$ such that $L \subseteq L(R)$.

Proof. For any given $L \in \mathscr{L}$, let us choose a ring $A$ such that $L \cong L(A)$ and set

$$
B=\bigoplus_{\mathscr{M} \in \operatorname{Max} A} A / \mathscr{M}
$$

let us consider the idealization $R=A(+) B$. We have $T(R)=R$ (see the proof of Theorem 1.7), and so $R \in \mathscr{R} \mathscr{C}$; moreover

$$
L(R) \supseteq\{I(+) B: I \text { ideal of } A\} \cong L(A)
$$

as desired.

It is not true that $\{L(R): R \in \mathscr{R} \mathscr{C}\}=\mathscr{L}$. In fact we have

PROPOSITION 2.5. For all $R \in \mathscr{R} \mathscr{C}$ we have $L(R) \not L(\mathbb{Z})$.

PROOF. If $R \in \mathscr{R} \mathscr{C}$ is an integral domain, then $R$ is a valuation domain, hence $L(R)$ is a chain and cannot be isomorphic to $L(\mathbb{Z})$. Thus we can assume that $Z(R) \neq\{0\}$; let $x, y \in R \backslash\{0\}$ be such that $x y=0$. If $R x \cap R y=0$ then $L(R) \not L(\mathbb{Z})$, since $L(\mathbb{Z})$ does not contain two nonzero elements whose inf is zero. On the other hand, if $0 \neq u \in R x \cap R y$, then $u^{2}=0$, so that the nilradical of $R$ is not zero. We infer that in $L(R)$ the intersection of all maximal elements is nonzero, which does not happen in $L(\mathbb{Z})$. 
In view of Proposition 2.4, it is natural to confine our attention to regular ideals of rings in $\mathscr{R} \mathscr{C}$. The following easy characterization extends an analogous result for valuation domains.

PROPOSITION 2.6. A ring $R$ lies in $\mathscr{R} \mathscr{C}$ if and only if the set of ideals of $R$ generated by regular elements is totally ordered by inclusion.

PROOF. Let us suppose that $I, J$ are ideals of $R \in \mathscr{R} \mathscr{C}$ generated by regular elements; then $I=\left(r_{\lambda}: \lambda\right), J=\left(s_{\mu}: \mu\right)$, with $r_{\lambda}, s_{\mu} \in \operatorname{Reg} R$, for all $\lambda, \mu$. Let us prove that $I \nsubseteq J$ implies $I \supseteq J$. In fact, choose $r_{\lambda_{0}} \in I \backslash J$; then $s_{\mu}$ does not divide $r_{\lambda_{0}}$ for all $\mu$, and therefore $r_{\lambda_{0}}$ divides all the $s_{\mu}$, so that $I \supseteq r_{\lambda_{0}} R \supseteq J$. This proves half of the statement. The converse is obvious.

Of course, a regular ideal is not necessarily generated by regular elements. Our next step is to investigate the rings $R$ such that the lattice of their regular ideals is a chain; we shall be led to a characterization of Prüfer $V$-valuated rings.

We recall that Anderson and Pascual in [2] extensively investigated sublattices of regular ideals of commutative rings; here we focus our attention to rings lying in $\mathscr{R} \mathscr{C}$.

For a given ring $R$, we shall denote by $L_{\text {reg }}(R)$ the sublattice of $L(R)$ formed by the regular ideals of $R$ (it is immediate to check that $L_{r e g}(R)$ is in fact a sublattice; note that it is not complete, in general).

As premised, we are interested in the case when $L_{\text {reg }}(R)$ is a chain. From Proposition 2.6 it follows that $R$ lies in $\mathscr{R} \mathscr{C}$ if $L_{\text {reg }}(R)$ is a chain.

LEMMA 2.7. Let $R$ be a ring; $L_{r e g}(R)$ is a chain if and only if, for all $r, s \in \operatorname{Reg} R$ and for all $x, y \in Z(R)$, the ideals $(r, x)$ and $(s, y)$ are comparable by inclusion.

PROOF. One implication is obvious. Let us now choose arbitrary regular ideals $I$, $J$ of $R$, and let us verify that they are comparable by inclusion. If $I \nsubseteq J$, there must exist a pair of elements $r, x \in I$, with $r$ regular and $x \in Z(R)$, such that $(r, x) \nsubseteq J$ (otherwise $J$ should contain a system of generators of $I$ ). But then, for every pair of elements $s, y \in J$ with $s$ regular and $y \in Z(R)$, we have $(r, x) \nsubseteq(s, y)$, whence $(r, x) \supseteq(s, y)$, by hypothesis. From the arbitrariness of the choice of $s, y$ we deduce that

$$
J \subseteq(r, x) \subseteq I
$$

from which the assertion.

LEMMA 2.8. If $L_{r e g}(R)$ is a chain, then every finitely generated regular ideal of $R$ is generated by two elements. 
PROOF. Let $J$ be a finitely generated regular ideal of $R$; since $R \in \mathscr{R} \mathscr{C}$, we readily see that $J$ can be written in the form

$$
J=\left(r, x_{1}, \ldots, x_{n}\right) \quad \text { with } r \in \operatorname{Reg} R \text { and } x_{i} \in Z(R) .
$$

In view of Lemma 2.7, we can assume that $\left(r, x_{1}\right) \supseteq\left(r, x_{n}\right)$, so that $J=\left(r, x_{1}, \ldots, x_{n-1}\right)$; by iterated applications of Lemma 2.7 we obtain that $J$ is two-generated, as desired.

The equivalence of (i) and (iii) in the next theorem was proved by Anderson and Pascual [2, Theorem 3.8]; however, here we give a complete proof of our result.

THEOREM 2.9. Let $R$ be a ring; the following are equivalent:

(i) $L_{\text {reg }}(R)$ is a chain;

(ii) $R$ is a Prüfer ring and $R$ is $V$-valuated;

(iii) $R$ is a Prüfer valuation ring.

ProOF. (i) $\Rightarrow$ (ii) Let us first show that $R$ is a Prüfer ring; in view of Lemma 2.8, it is enough to prove that every ideal of the form $J=(r, z)$, with $r \in \operatorname{Reg} R$, is invertible. If $z \in r R$, then obviously $J=r R$ is invertible. Thus we can assume that $z \notin r R$ and also that $z \in Z(R)$, otherwise $J$ is again a principal ideal, since $R \in \mathscr{R} \mathscr{C}$. Let us compare the regular ideals $r R$ and $\left(r^{2}, z\right)$; from $z \notin r R$ it follows $\left(r^{2}, z\right) \supseteq r R$, and therefore

$$
r=\alpha r^{2}+\beta z \quad \text { for suitable } \alpha, \beta \in R .
$$

Then we have

$$
1=\alpha r+(\beta / r) z
$$

where $\alpha \in R \subseteq(r, z)^{-1}=J^{-1}$ and $\beta / r \in J^{-1}$, since $(\beta / r) r=\beta \in R$ and $(\beta / r) z=1-\alpha r \in R$. We conclude that $J J^{-1}=R$, that is $J$ is invertible, as desired.

Let us now verify that $R$ is $V$-valuated. By contradiction, let us suppose that there exist $x, y \in R$ and $r \in \operatorname{Reg} R$ such that $x / r, y / r \in T(R) \backslash R$ and $(x / r)(y / r) \in R$. Since $L_{r e g}(R)$ is a chain, we can assume without loss that $(x, r) \supseteq(y, r)$ and so

$$
y=\alpha x+\beta r \quad \text { for suitable } \alpha, \beta \in R
$$

from which

$$
y / r=\alpha x / r+\beta .
$$

Let $\xi=\alpha x / r$; we have $\xi \notin R$, since $y / r \notin R$; moreover $\xi y / r \in \alpha R \subseteq R$. From $y / r=\xi+\beta$ we deduce the following relation

$$
\xi(\xi+\beta) \in R \quad \text { with } \xi \in T(R) \backslash R .
$$


But (3) is impossible, since $R$ is integrally closed, being a Prüfer ring. From the contradiction we get the desired conclusion.

(ii) $\Rightarrow$ (i) In view of Lemma 2.7 it is enough to verify that any two ideals of the form $(r, x),(s, y)$ with $r, s \in \operatorname{Reg} R$ and $x, y \in Z(R)$ are comparable by inclusion. Let $v$ be the $V$-valuation defined on $T=T(R)$; we can assume, without loss, that $T \neq R$ (otherwise the result is trivial) and that $v(y) \geq v(x)$. Since $(x, r)$ is invertible, we have the relation

$$
1=\xi x+\eta r \quad \text { with } \xi, \eta \in(x, r)^{-1}
$$

from (4) we obtain

$$
y=(\xi y) x+(\eta y) r .
$$

Now, by the definition of $v$, from $v(y) \geq v(x)$ we get $(R: y) \supseteq(R: x) \supseteq(x, r)^{-1}$; we infer that $\xi y, \eta y \in R$, whence $y \in(x, r)$. Let us now examine $s \in \operatorname{Reg} R$. If $s / r \in R$ we have $s \in(x, r)$, whence $(x, r) \supseteq(y, s)$. If $r / s \in R$ and $v(s)<v(x)$, we have $x / s \in R$, whence $(x, r) \subseteq s R \subseteq(y, s)$. Finally, if $v(s) \geq v(x)$, arguing as above with $s$ in the place of $y$, we get $s \in(x, r)$ and so $(y, s) \subseteq(x, r)$. Therefore, in any case, we have the desired conclusion.

(iii) $\Rightarrow$ (ii) Trivial.

(i) and (ii) $\Rightarrow$ (iii) Let $v$ be the $V$-valuation defined on $T$; let us consider the prime ideal of $R$,

$$
P_{v}=\{x \in T: v(x)>0\} .
$$

We shall prove that $R$ is a Manis valuation ring, via [7, Theorem 5.1]: we must verify that, for all $\xi \in T \backslash R$ there exists $\alpha \in P_{v}$ such that $\xi \alpha \in R \backslash P_{v}$. Let us write $\xi=y / r$, with $y \in R, r \in \operatorname{Reg} R \backslash U(R)$. Since $L_{r e g}(R)$ is a chain, the regular ideals $r R$ and $\left(y, r^{2}\right)$ are comparable by inclusion. On the other hand, $y \notin r R$, since $\xi \notin R$ and therefore $r \in\left(y, r^{2}\right)$, so that

$$
r=\alpha y+\beta r^{2} \quad \text { with } \alpha, \beta \in R
$$

whence

$$
1=\alpha y / r+\beta r .
$$

We deduce that $\alpha \xi=1-\beta r \in R \backslash P_{v}$, since $v(r)>0$ implies $\beta r \in P_{v}$. Let us show that $\alpha \in P_{v}$, that is $v(\alpha)>0$, whence our conclusion. In fact, $v(\alpha)=0$ would imply

$$
v(\xi)=v(\xi)+v(\alpha)=v(\xi \alpha)=0
$$

so that $\xi \in R$, which is impossible.

The implication (ii) $\Rightarrow$ (iii) of the above theorem is, in some sense, related to [7, Theorem 7.7]; there, it is proved that a Marot ring in $\mathscr{R} \mathscr{C}$ is automatically a Manis valuation ring; here we show that a Prüfer ring in $V$-val is automatically in $\mathscr{M} \mathscr{V} \mathscr{R}$. 
If a ring $R$ is $V$-valuated by $v$, the counterimage $v^{-1}(\infty)$ of $\infty \in \Gamma$ is an ideal, which is prime since $\alpha, \beta<\infty$ implies $\alpha+\beta<\infty$ for all $\alpha, \beta \in \Gamma$ [4, Lemma 2.5]. It is natural to investigate the analogy of the ideal $v^{-1}(\infty)$ in any ring $R \in \mathscr{R} \mathscr{C}$.

As remarked in the first section, the case when $R=T(R)$ constitutes an exception, due to the fact that $R$ does not determine its valuation. Thus in this section we shall always assume that $R \neq T(R)$.

For an assigned $R \in \mathscr{R} \mathscr{C}, R \neq T(R)$, we define

$$
I_{\infty}=\{x \in R: x / r \in R \text { for all } r \in \operatorname{Reg} R\} .
$$

It is routine to check that $I_{\infty}$ is an ideal of $R$ and that $I_{\infty} \subseteq Z(R)$, since $R \neq T(R)$; moreover $I_{\infty}=\bigcap_{r \in \operatorname{Reg} R} r R$. We also note that $I_{\infty}$ is an ideal of $T(R)$, too, as an obvious consequence of the fact that $x \in I_{\infty}$ implies $x / r \in I_{\infty}$, for all $r \in \operatorname{Reg} R$.

We shall write $I_{\infty}(R)$ when we want to emphasize the dependence on the ring $R$.

Proposition 3.1. Let $R \neq T(R)$ be a $V$-valuated ring; then $I_{\infty}=v^{-1}(\infty)$, where $v$ is the $V$-valuation determined by $R$.

PROOF. From $v(x y)=v(x)+v(y)$, for $x, y \in T(R)$, and $\gamma+\infty=\infty$, for $\gamma \in \Gamma$, it follows that $v^{-1}(\infty) \subseteq I_{\infty}$. Conversely, let $x \in I_{\infty}$ and assume, by contradiction, that $v(x)<\infty$; by Proposition 1.4, there exists $r \in \operatorname{Reg} R$ such that $v(x)<v(r)$, and therefore $x / r \notin R$, against the definition of $I_{\infty}$.

We recall that a ring $R$ is said to be chained if its ideals are totally ordered by inclusion, that is $L(R)$ is a chain (see $\$ 23$ of [7]).

PROPOSITION 3.2. Let $R$ be a ring in $\mathscr{R} \mathscr{C}$.

(i) If $I_{\infty}=Z(R)$, then $R$ is a local Manis valuation ring;

(ii) if $R / I_{\infty}$ is chained, then $R$ is a Manis valuation ring and $I_{\infty}$ is a prime ideal.

PROOF. (i) Let $J$ be the ideal generated by $\operatorname{Reg} R \backslash U(R) ; J$ is proper by Lemma 1.1. It is clear that $J \supseteq I_{\infty}=Z(R)$, so that any $u \in R \backslash J$ is a unit, whence $R$ is a local ring with unique maximal ideal $J$. To prove that $R$ is a Manis valuation ring, we again make use of [7, Theorem 5.1]. Choose $\xi=x / r \in T(R) \backslash R$; from $I_{\infty}=Z(R)$ it follows that $x \notin Z(R)$. Since $R \in \mathscr{R} \mathscr{C}$, we can assume that $\xi=1 / r$, with $r \in J$, and thus we have $r \xi=1 \in R \backslash J$, as desired.

(ii) Since $R / I_{\infty}$ is chained, and each regular ideal of $R$ contains $I_{\infty}, L_{r e g}(R)$ will be a chain. From Theorem 2.9 we obtain that $R$ is a Prüfer Manis valuation ring, whence $I_{\infty}$ is a prime ideal, by Proposition 3.1. 
Looking at point (i) of the above proposition, it is natural to ask if $R \in \mathscr{R} \mathscr{C}$ and $R$ local is enough to ensure that $R$ be a Manis valuation ring. This is not true in general, as we shall see in the following example.

EXAMPLE 3.3. We consider an adaptation of the ring constructed in the proof of Theorem 1.7. In that same notation, let $D=A+P x+x^{2} A[x]$ and let $\mathscr{M}=$ $P+P x+x^{2} A[x] ; \mathscr{M}$ is a maximal ideal of $D$; we consider the localization $D_{\mathscr{M}}$. Let $B=\bigoplus_{f \in \mathscr{U}} Q[x] /(f)$, where $\mathscr{U}=\{f \in Q[x] \mid(f) \cap D \subseteq \mathscr{M}$ and $f$ is irreducible $\}$. Then the idealization $R_{0}=D_{\mathscr{M}}(+) B$ is a local ring, with maximal ideal $\mathscr{M}(+) B$. It is easy to check that $\mathscr{X}_{D_{M}}=A_{P} \backslash\{0\}$, and arguments similar to those used to establish Theorem 1.7 will verify that $R_{0} \in \mathscr{R} \mathscr{C} \backslash V$-val.

We know that $I_{\infty}$ is a prime ideal if $R$ is $V$-valuated. It is interesting to observe that this fact is not true for a general $R \in \mathscr{R} \mathscr{C}$; this will be shown in the subsequent Example 3.5.

Though $I_{\infty}$ is not in general a prime ideal, yet some properties, enjoyed by prime ideals, hold for it.

PROPOSITION 3.4. Let $R \neq T(R)$ be a ring in $\mathscr{R} \mathscr{C}$.

(i) If $r x \in I_{\infty}$, where $r \in \operatorname{Reg} R$, then $x \in I_{\infty}$;

(ii) if $x^{2}=0$, then $x \in I_{\infty}$;

(iii) if $x y=0$, where Ann $x \cap$ Ann $y=0$, then either $x$ or $y$ are in $I_{\infty}$; in particular, if $e \in R$ is idempotent then either $e$ or $1-e$ are in $I_{\infty}$.

PROOF. (i) Follows at once from the definition of $I_{\infty}$.

(ii) Let $x \in R$ be such that $x^{2}=0$. Choose an arbitrary $r \in \operatorname{Reg} R$; then from $\left(x+r^{2}\right)\left(x-r^{2}\right)=-r^{4} \in \operatorname{Reg} R$ it follows that $x+r^{2} \in \operatorname{Reg} R$. Let us compare the regular elements $r$ and $x+r^{2}$ : if $x+r^{2}=r s$, we have $x \in r R$; if $\left(x+r^{2}\right) s=r$ we have $r x=r^{2} s x$, whence $x \in r R$, again. From the arbitrariness of $r \in \operatorname{Reg} R$ we get $x \in \bigcap_{r \in \operatorname{Reg} R} r R=I_{\infty}$.

(iii) We first observe that, if $x y=0$ and Ann $x \cap$ Ann $y=0$, then $r x+s y$ is regular for all $r, s \in \operatorname{Reg} R$. In fact $(r x+s y) z=0$ implies $r x z=-s y z$; since $r x z \in$ Ann $y$ and $s y z \in$ Ann $x$, we must have $x z=0=y z$, so that $z \in$ Ann $x \cap$ Anny $=0$.

Let us now suppose that $x \notin I_{\infty}$ and prove that then $y \in I_{\infty}$. Since the regular elements are comparable, it suffices to prove that $x / r \notin R$ implies that $y / r \in R$, for any assigned $r \in \operatorname{Reg} R$. In fact, let us compare the regular elements $r x+r^{2} y$ and $r^{2} x+r y ;$ if

$$
\left(r^{2} x+r y\right) s=r x+r^{2} y \quad \text { with } s \in \operatorname{Reg} R
$$

then $\left(r^{2}-r s\right) y=\left(r^{2} s-r\right) x \in \operatorname{Ann} x \cap$ Ann $y$, whence $r x=r^{2} s x$ and $x=r s x \in r R$, against our assumption. Thus, necessarily,

$$
\left(r x+r^{2} y\right) s=r^{2} x+r y
$$


from which, arguing as above, we get $y=r s y \in r R$, as desired.

Finally, we note that Anne $\cap \operatorname{Ann}(1-e)=0$ if $e$ is idempotent.

As promised, we finish showing that $I_{\infty}$ can be a non-prime ideal.

EXAMPLE 3.5. Let $A$ be a valuation domain, with maximal ideal $P$ and field of quotients $Q$, and let $x, y$ be indeterminates. Let $D=A[x, y]$, let $\mathscr{U}$ be the set of prime ideals of $Q[x, y]$ and set $B=\bigoplus_{\mathscr{P} \in \mathscr{U}} Q[x, y] / \mathscr{P}$.

We consider the idealization $R=D(+) B$ and the ideal $J=x y R=x y D(+) x y B$ of $R$; let $R_{1}=R / J$. We shall verify that $R_{1} \in \mathscr{R} \mathscr{C}$ and that the ideal $I_{\infty}\left(R_{1}\right)$ of $R_{1}$ is not prime.

In order to prove that $R_{1} \in \mathscr{R} \mathscr{C}$, we first prove the following

CLAIM. $\alpha=(f, b)+J \in R_{1}$ is regular if and only if $f \equiv c(\bmod x y D)$, for a suitable $0 \neq c \in A$.

PROOF. ( $\Longleftarrow)$ Let $\beta=\left(g, b^{\prime}\right)+J \in R_{1}$ be such that $\alpha \beta=0$. Then the following relations must hold:

$$
\begin{gathered}
f g \equiv 0 \quad \bmod x y D \\
f b^{\prime}+g b \in x y B .
\end{gathered}
$$

From (6) we get $c g \in x y D$, so that $g \in x y D$, since $c \neq 0$; therefore (7) reduces to $c b^{\prime} \in x y B$, which implies $b^{\prime} \in x y B$. This means that $\beta=0$, whence we conclude that $\alpha$ is regular.

$(\Longrightarrow)$ We can assume that $f \notin A$, otherwise the assertion is trivial. Let us write $f$ as a product of irreducible elements of $Q[x, y], f=\prod_{j} g_{j}$. Now observe that, for every $j$,

$$
(f, b)\left(0,1+g_{j} Q[x, y]\right)=0 \in J
$$

so that $\alpha$ regular implies

$$
1+g_{j} Q[x, y] \in x y B \text { for all } j
$$

(recall that $g_{j} Q[x, y]$ is a prime ideal, since $g_{j}$ is irreducible). From $f=\prod_{j} g_{j}$ and the relation (8) we obtain the following congruence of polynomials

$$
f f_{1} \equiv 1 \bmod x y Q[x, y]
$$

for $f_{1} \in Q[x, y]$ conveniently chosen. Let

$$
f \equiv c+x h(x)+y k(y), \quad f_{1} \equiv c_{1}+x h_{1}(x)+y k_{1}(y) \quad \bmod x y Q[x, y] .
$$

By direct computation we see that (9) implies $h(x)=k(y)=0$ and $c \neq 0$, whence $f \equiv c \bmod x y Q[x, y]$; finally, $f \in D$ implies $c \in A$ and $f \equiv c \bmod x y D$, as desired. 
In view of the claim, to establish that $R_{1} \in \mathscr{R} \mathscr{C}$ it is enough to verify that $\alpha=$ $(c, b)+J$ and $\alpha^{\prime}=\left(c^{\prime}, b^{\prime}\right)+J$, where $c, c^{\prime} \in A \backslash\{0\}$, are always comparable. Let us assume, without loss, that $c^{\prime}=c t$, with $t \in A$; from the equality in $R$,

$$
(c, b)\left(t, c^{-1}\left(b^{\prime}-b t\right)\right)=\left(c^{\prime}, b^{\prime}\right)
$$

it follows at once that $\alpha$ divides $\alpha^{\prime}$ in $R_{1}$, as desired.

It remains to show that $I_{\infty}\left(R_{1}\right)$ is not a prime ideal. We fix $0 \neq p \in P$; now $x+J, y+J \in R_{1}$ and their product is zero; to conclude, we will show that $p+J \in$ $\operatorname{Reg} R_{1}$ divides in $R_{1}$ neither $x+J$ nor $y+J$, so that they both do not lie in $I_{\infty}\left(R_{1}\right)$. In fact, the relation

$$
(p+J)((f, b)+J)=x+J \quad \text { with }(f, b) \in R
$$

gives rise to the congruence

$$
p f-x \equiv 0 \quad \bmod x y D
$$

which cannot hold, since the coefficient of $x$ in the first member of (10) cannot disappear. In a similar way we argue for $y+J$.

All our statements are thus verified.

\section{References}

[1] J. Alajbegovic and P. Zanardo, 'Uniserial modules over manis rings', Comm. Algebra 17 (1989), 1463-1476.

[2] D. D. Anderson and J. Pascual, 'Regular ideals in commutative rings, sublattices of regular ideals, and Prüfer rings', J. Algebra 111 (1987), 404-426.

[3] J. Gräter, 'An integrally closed ring which is not the intersection of valuation rings', Proc. Amer. Math. Soc. 107 (1989), 333-336.

[4] D. K. Harrison and M. A. Vitulli, 'V-valuations of a commutative ring', J. Algebra 126 (1989), 264-292.

[5] —_, 'Complex-valued places and cmc subsets of a field', Comm. Algebra 17 (1989), 2529-2537.

[6] - 'A categorical approach to the theory of equations', J. Pure Appl. Algebra 67 (1990), 15-31.

[7] J. A. Huckaba, Commutative rings with zero divisors (Marcel Dekker, New York, 1988).

[8] T. G. Lucas, 'Valuation rings and integral closure', Canad. Math. Bull. 33 (1990), 327-330.

[9] W. Krull, 'Allgemeine Bewertungstheorie', J. Reine Angew. Math. 167 (1932), 160-196.

[10] M. Manis, 'Valuations on a commutative ring', Proc. Amer. Math. Soc. 20 (1969), 193-198.

[11] D. A. Marcus, Number fields (Springer, New York, 1977).

[12] J. Marot, 'Extension de la notion d'anneau valuation', Dept. Math. Faculté Sci. de Brest (1968).

[13] M. Nagata, Local rings (Wiley, Interscience, New York, 1962).

[14] P. Samuel, 'La notion de place dans un anneau', Bull. Soc. Math. France 85 (1957), 123-133.

[15] J. H. Silverman, The arithmetic of elliptic curves (Springer, New York, 1986).

[16] P. Zanardo, 'Constructions of Manis valuation rings', Comm. Algebra 21 (1993), 4183-4194. 
Dipartimento di Matematica Pura e Applicata

Via Belzoni 7

35131 Padova

Italy

e-mail: pzanardo@pdmat1.unipd.it 